\title{
Bioinspired wood-like coaxial fibers based on MXene@graphene oxide with superior mechanical and electrical properties
}

\author{
Chen, Xing; Jiang, Jianjun; Yang, Guoyu; Li, Chuanbing; Li, Yujun
}

Published in:

Nanoscale

Link to article, DOI:

10.1039/d0nr04928

Publication date:

2020

Document Version

Peer reviewed version

Link back to DTU Orbit

Citation (APA):

Chen, X., Jiang, J., Yang, G., Li, C., \& Li, Y. (2020). Bioinspired wood-like coaxial fibers based on

MXene@graphene oxide with superior mechanical and electrical properties. Nanoscale, 12(41), 21325-21333.

https://doi.org/10.1039/d0nr04928j

\section{General rights}

Copyright and moral rights for the publications made accessible in the public portal are retained by the authors and/or other copyright owners and it is a condition of accessing publications that users recognise and abide by the legal requirements associated with these rights.

- Users may download and print one copy of any publication from the public portal for the purpose of private study or research.

- You may not further distribute the material or use it for any profit-making activity or commercial gain

- You may freely distribute the URL identifying the publication in the public portal 


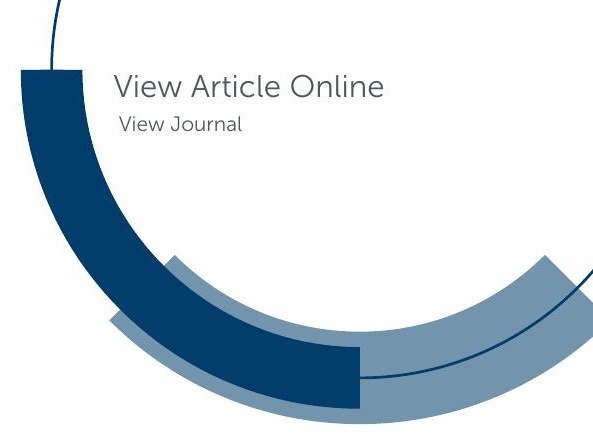

\section{Accepted Manuscript}

This article can be cited before page numbers have been issued, to do this please use: X. Chen, J. Jiang,

G. Yang, C. Li and Y. Li, Nanoscale, 2020, DOI: 10.1039/D0NR04928J.
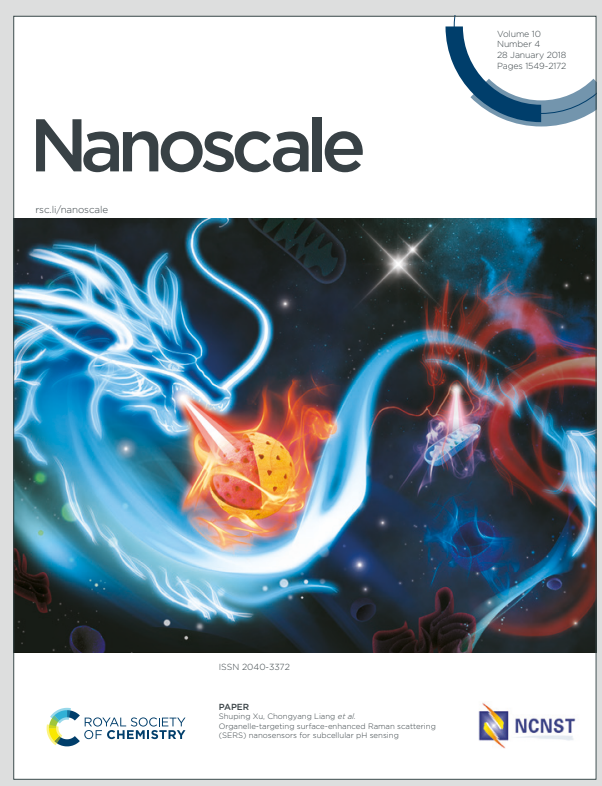

This is an Accepted Manuscript, which has been through the Royal Society of Chemistry peer review process and has been accepted for publication.

Accepted Manuscripts are published online shortly after acceptance, before technical editing, formatting and proof reading. Using this free service, authors can make their results available to the community, in citable form, before we publish the edited article. We will replace this Accepted Manuscript with the edited and formatted Advance Article as soon as it is available.

You can find more information about Accepted Manuscripts in the Information for Authors.

Please note that technical editing may introduce minor changes to the text and/or graphics, which may alter content. The journal's standard Terms \& Conditions and the Ethical guidelines still apply. In no event shall the Royal Society of Chemistry be held responsible for any errors or omissions in this Accepted Manuscript or any consequences arising from the use of any information it contains. 
Bioinspired wood-like coaxial fibers based on MXene@graphene oxide withl: superior mechanical and electrical properties

Xing Chen, ${ }^{1,2}$ Jianjun Jiang, ${ }^{1 *}$ Guoyu Yang, ${ }^{1}$ Chuanbing Li, ${ }^{1}$ Yujun $\mathrm{Li}^{1 *}$

${ }^{1}$ Shaanxi Engineering Research Center for Digital Manufacturing Technology, Northwestern Polytechnical University, Xi'an 710072, P. R China

${ }^{2}$ Department of Environmental Engineering, Technical University of Denmark, Miljøvej 113, DK-2800 Kongens Lyngby, Denmark

E-mail: jianjun@nwpu.edu.cn(J. Jiang); li.yujun@nwpu.edu.cn(Y.Li)

KEYWORDS : MXenes, Graphene, Biomimetic core-shell fiber, Nano-structures, Mechanical property 


\begin{abstract}
MXenes, a new class of two-dimensional materials with excellent performance, are promising materials for wearable energy storage devices. However, the lack of sufficient interaction between various MXene particles makes it difficult to translate the exceptional performance from nanoscale to macroscale. Additionally, the intrinsic characteristic of easy oxidation limits their practical applications. Herein, inspired by the structure of wood, a biomimetic core-shell MXene@graphene oxide (MX@GO) fiber was fabricated using GO as a mechanical layer to wrap MXenes. The GO layer could easily assemble MXene particles into macroscale materials and protected them from oxidation. Therefore, the as-fabricated core-shell MX@GO fiber showed a high tensile strength $(290 \mathrm{MPa})$ and excellent electrical conductivity $(2400 \mathrm{~S} / \mathrm{m})$. Notably, the conductivity of biomimetic fiber only decreased to $1800 \mathrm{~S} / \mathrm{m}$ with a reduction of about $30 \%$ at $100^{\circ} \mathrm{C}$. This work paves the way to fabricate MXene-based fiber with freely designed functionalities and morphologies, which are suitable for various high-value fabricbased applications.
\end{abstract}

\title{
1. Introduction
}

The pursuit of eco-friendly and energy-efficient technologies highlights the increasing demands for lightweight and multifunctional materials with excellent mechanical performance. Benefitting from extraordinary electrochemical and structural properties, MXenes have shown a grand promise for applications in energy storages, electromagnetic interference shielding, and oxygen evolution reactions since their discovery in 2011.[1-4] Unfortunately, the lack of sufficient interaction between various MXene particles makes it difficult to translate the exceptional performance from the nanoscale to the macroscale bulk materials.[5] Moreover, the easily oxidized surface reduces their electrical conductivity. These issues limit their practical applications. To overcome these shortcomings, several researchers have recently attempted to construct the MXene microstructure by introducing GO, bacterial cellulose, polyimide, etc.[5-7] For instance, Ma et al. [6] designed MXene/reduced graphene oxide (rGO) 

aerogel. Chen et al. [5] fabricated a lightweight carbon aerogel with both exceptionally high mechanical and sensing performances using bacterial cellulose as a nanobinder to join MXene $\left(\mathrm{Ti}_{3} \mathrm{C}_{2}\right)$ nanosheets into a lamellar macrostructure. Although the macroscale properties of the obtained 3D MXene composites could be improved by mixing different particles, the introduction of nanoparticles may block the electron transport paths between MXene.

In nature, the evolutionary selection pressure has promoted animal and plants to develop abilities to tightly control their microstructure with well-ordered arrangements at the nano- and molecular level, which endows natural structures with unique properties.[8] Recently, inspired by nature, various high-performance materials were fabricated, such as a nacre-like structure[9], bio-inspired polymeric woods[10] and lotus-leaf-like superhydrophobic surface[11]. Remarkably, woods have been widely used in engineering due to its unique combination of lightness and mechanical efficiency. The wood cell wall is mainly composed of a primary and three secondary layers (S1, S2 and S3). Within the secondary layer, the outer cell wall layer (S2 layer), made of the aligned cellulose microfibrils, has the highest modulus and tensile strength among all layers. Cellulose microfibrils orientation not only controls passive mechanical properties but also regulates the ability of wood cells to generate stress. Meanwhile, the inner cell wall layer (S3 layer), made of the disordered cellulose microfibrils, is responsible for the transportation of water and other nutrients.[12, 13] The S2 and S3 layers are found to serve as a mechanical and functional layer, respectively, which gives rise to its superior performance. The microstructural features of the cell wall in wood provide a hint that it may be possible to construct an MXene-based fiber with a mechanical support layer and a functional layer. In the fiber, MXenes can perform its inherent electrochemical properties directly as a core layer.

Due to the weak interaction between MXene particles, the material used for the mechanical layer should have the excellent gelation ability to assemble MXenes into a macro fiber and the unique mechanical properties to provide the MXene-based fiber with remarkable performance. With the high strength, excellent thermal conductivity and good electrical 
conductivity, graphene has a wide range of applications in flexible transparen€alectrodesterico4928J multifunctional composites since its discovery in 2004.[14] Compared with graphene, GO possesses many oxygen-containing functional groups, including carboxyl, hydroxyl, epoxy groups, etc. on their basal edges and planes[15], which gives rise to its excellent gelation ability. Gao's group[16] first fabricated neat graphene fiber from concentrated GO liquid crystal by wet-spinning methodology, which shown exceptional mechanical and electrical properties than composite fiber.[17] Therefore, GO nanosheets are an ideal material as the mechanical support layer to warp MXenes.

Herein, inspired by the structure of wood, we demonstrate the delicate design and synthesis of a wood-like coaxial MX@GO fiber with a superior mechanical shell and an electrical core. The advantage of good gelation ability of GO can be brought into full play to assemble MXene sheets into the macro fiber. Still, the problem of easy surface oxidation of MXene sheets can be considered to obtain a high-performance fiber. The obtained fiber shows both high tensile strength and electrical conductivity. Moreover, the conductivity of biomimetic fiber remains stable with the increase in temperature. These advantages allow the bioinspired wood-like coaxial fiber to have potential applications in flexible wearable energy devices.

\section{Experimental}

\subsection{Material}

The used $\mathrm{Ti}_{3} \mathrm{AlC}_{2}$ powders were purchased from Jilin 11 technology Co., Ltd. (Jilin, China). Lithium fluoride (LiF), Crystalline flake graphite (purity $>99.9 \%$ ), phosphoric acid $\left(\mathrm{H}_{3} \mathrm{PO}_{4}, 85 \%\right)$ and sodium ascorbate (purity $\left.>99.9 \%\right)$ were supplied by Aladdin Industrial Corporation (China). Concentrated sulfuric acid $\left(\mathrm{H}_{2} \mathrm{SO}_{4}, 98 \%\right)$, potassium permanganate $\left(\mathrm{KMnO}_{4}\right)$, hydrogen peroxide $\left(\mathrm{H}_{2} \mathrm{O}_{2}, 38 \%\right)$, hydrochloric acid $(\mathrm{HCl}, 38 \%)$, Calcium chloride anhydrous $\left(\mathrm{CaCl}_{2}\right.$, purity $\left.>96 \%\right)$, hydroiodic acid ( $\left.\mathrm{HI}, \geqslant 45 \%\right)$ and Ethanol (purity $>99 \%$ ) were purchased from Sinopharm Chemical Reagent CO., Ltd. (China) and used without further purification.

\subsection{Synthesis of Graphene oxide and $\mathrm{Ti}_{3} \mathrm{C}_{2} \mathrm{~T}_{\mathrm{x}}$}

The GO and MXene were prepared according to the reported method.[15, 18] 


\subsection{Preparation of MX/GO hybrid fiber}

A given amount of MXene sheets was added to the GO dispersion. The mixture was kept at room temperature under agitation for about $10 \mathrm{~min}$. The MXene/GO solution was then loaded into a plastic syringe and extruded into a coagulation bath by an injection pump (0.3 $\mathrm{ml} / \mathrm{min})$. The coagulation bath was a mixture of Ethanol and DI water $(3: 1 \mathrm{wt} \%)$ with $5 \mathrm{wt} \%$ $\mathrm{CaCl}_{2}$. After coagulation, the fiber was transferred into the water bath to wash away the residual coagulation solution, and the washed MX/GO fiber was collected onto a reel.

\subsection{Preparation of MX@GO coaxial fiber}

The coaxial wet-spinning setup was home-made, which consisted of two syringes and a spinneret with an inner diameter of $0.26 \mathrm{~mm}$ needle $(25 \mathrm{G})$ coaxially placed inside an inner diameter of $1.12 \mathrm{~mm}$ one $(17 \mathrm{G})$. The feed rate of the inner and outer solution was set at 1 and $0.5 \mathrm{ml} / \mathrm{min}$, respectively. The coagulation was the same as before. After washing and drying, final MX@GO fiber was obtained.

The wood-like core-shell MX@GO-x (x represents the mass ratio of MXene to GO) fiber was fabricated with a mechanical support layer and a functional layer. The MX/GO-x hybrid fiber was manufactured using graphene sheets as a nanobinder to connect MXene with a unique setup (Fig. 1).

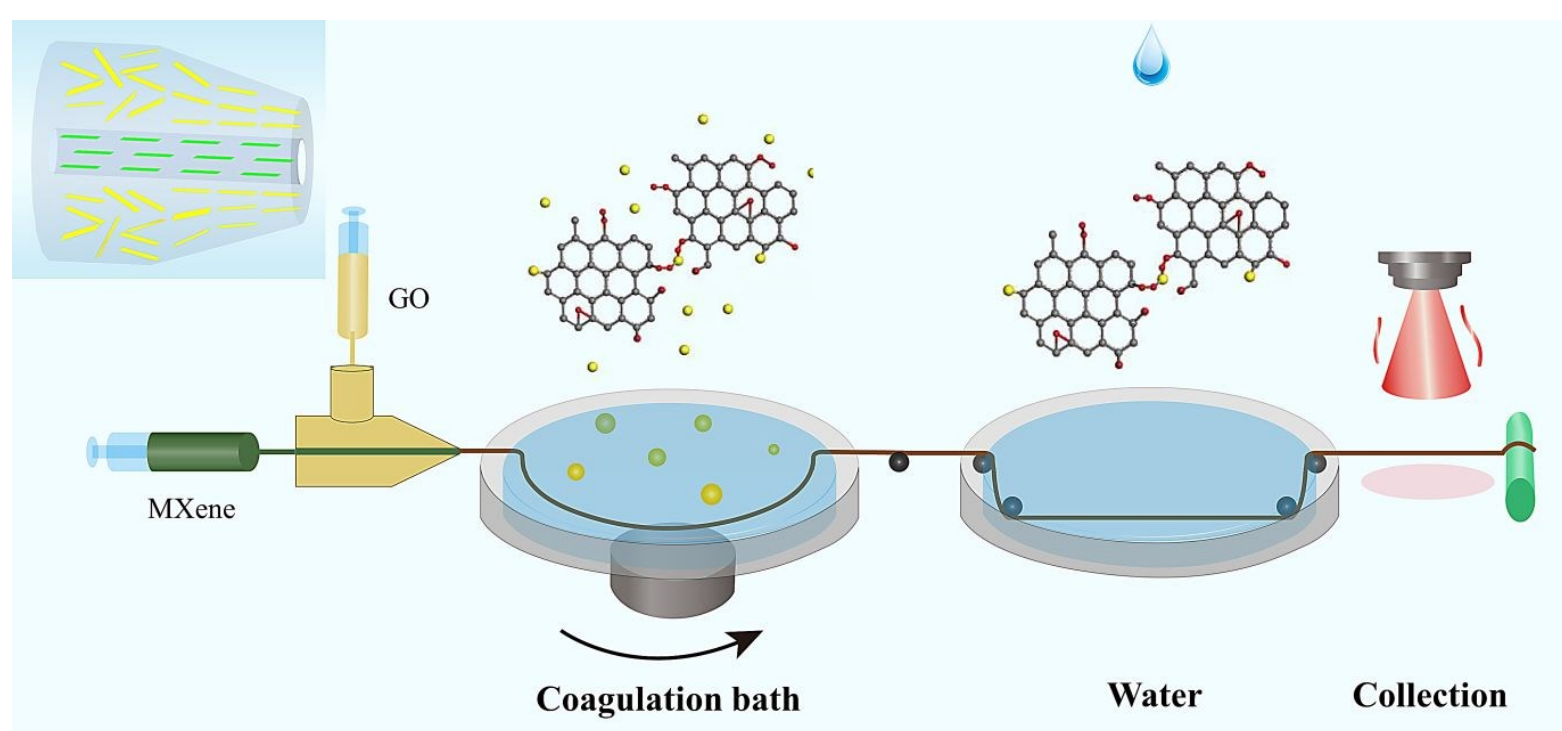

Fig. 1 The schematic illustration of the coaxial wet-spinning process for MX@GO fiber.

\subsection{Characterization.}

The morphology of obtained fibers was characterized by the field emission scanning 

acquired using energy-dispersive X-ray (EDX) spectroscopy. X-ray diffraction (XRD) patterns of GO and MXene were recorded with a X-ray diffractometer (BRUKER, D8 ADVANCE, Germany) using CuK $\alpha$ radiation $(\lambda=1.54 \AA)$ at a generator voltage of $40 \mathrm{kV}$ and a generator current of $40 \mathrm{~mA}$ with a scanning speed of $4 \%$ min. A Thermo Fisher K-Alpha X-ray photoelectron spectroscopy (XPS) was used to analyze the chemical compositions of GO. The qualities of GO was further scanned using a Raman Microscope(Renishaw, invia reflex, UK). The chemical structures of MXene was examined based on the Fourier transform infrared spectra (Nicolet, IS5) obtained. The mechanical property of fiber was evaluated by a tensile instrument (Shanghai New Fiber Instrument Co., Ltd, XQ-1C, China). Electrical measurements (I-V curves) were carried out on an electrochemical workstation (using a two-electrode setup) by measuring the current produced at different gate voltages (Princeton, PARSTAT3000A, USA). Conductivity values were calculated from the slopes of the I-V curves and the thickness of each film which was measured using a cross-sectional SEM. The electromagnetic parameters were measured by vector network analyzer (Agilent, PNA-N5244A) using the coaxial method.

\section{Results and discussion}

As shown in Fig. 2b, the GO and MXene dispersion are light brown and dark green, respectively. With the addition of MXene sheets into the stable suspension of GO in water, MXene sheets begin to disperse in GO solution, which appears blackish green. The graphitic carbon and oxidized carbon in the XPS spectra were calculated to be $35.5 \%$ and $64.5 \%$ (Fig. $\mathrm{S} 1$ ), respectively, which indicates that GO has comparable oxidization degrees. Raman spectra show D peaks $\sim 1590 \mathrm{~cm}^{-1}$ and $\mathrm{G}$ peaks $\sim 1350 \mathrm{~cm}^{-1}$, confirming the lattice distortions of graphene basal planes (Fig. S2). Only the $(00 l)$ peaks exist, and the peak at $39^{\circ}$ vanishes in the X-ray diffraction patterns of the as-fabricated MXene. Besides, the (002) peak shifts to a smaller angle, indicating the complete exfoliation of MXene (Fig. S3-4). The morphologies of the fiber were characterized by SEM techniques. Similar to GO fiber, the MX/GO fiber has a highly dense structure and well-aligned graphene sheets on fiber surfaces (Fig. 2i). The energy- 


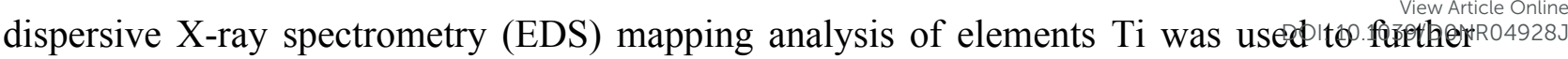
verify the dispersibility of MXene sheets in MX/GO fiber. The dominant Ti element well distinguishes this hybrid nanoscopic structure. It can be observed that Ti element is evenly dispersed in the whole system, as shown in Fig. 2f, which indicates the presence of GO nanosheets can entangle with each other to connect MXene nanosheets and reveal that MXene is well-distribution on GO sheets. Compared with MX/GO fiber, the core-shell MX@GO fiber is easily identified in Fig. 2d. The compact layered structures feature the MXene core and the GO sheath wraps the core tightly without any gaps and voids, which is extremely important for improving the mechanical properties of MX@GO fiber. As shown in elemental mapping analysis for Ti atoms (Fig. 2g), the hierarchical microstructure is well distinguished by dominant $\mathrm{Ti}$ atoms from the fiber cross-section, which proves the wood-like core-shell MX@GO fiber has been successfully produced. Therefore, the well-aligned GO shell may provide mechanical support for the MX@GO fiber, and the MXene core may be directly used as the electrical transfer path. More importantly, these fibers are highly oriented along the fiber axis (Fig. 2 h-j). 
a)
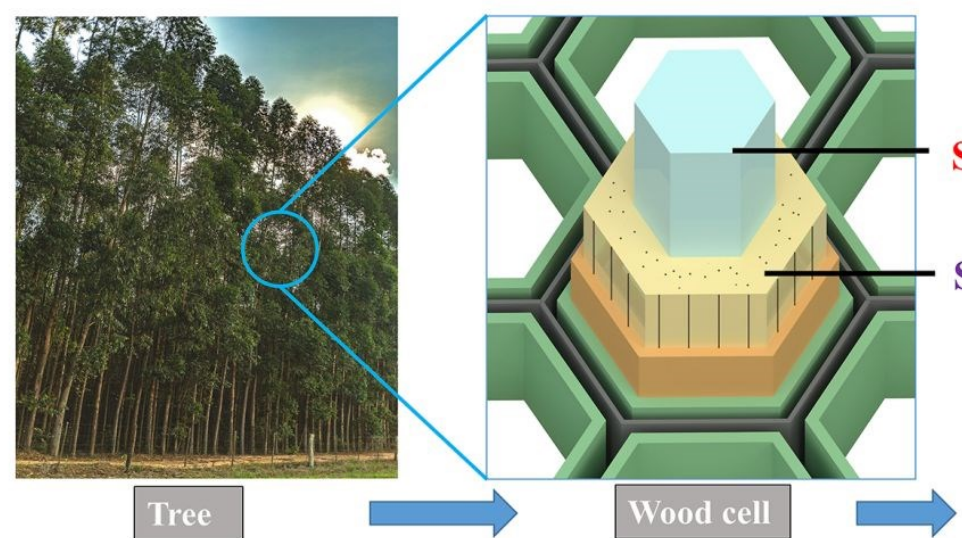

View Article Online DOI: $10.1039 / D 0 N R 04928 J$

b)
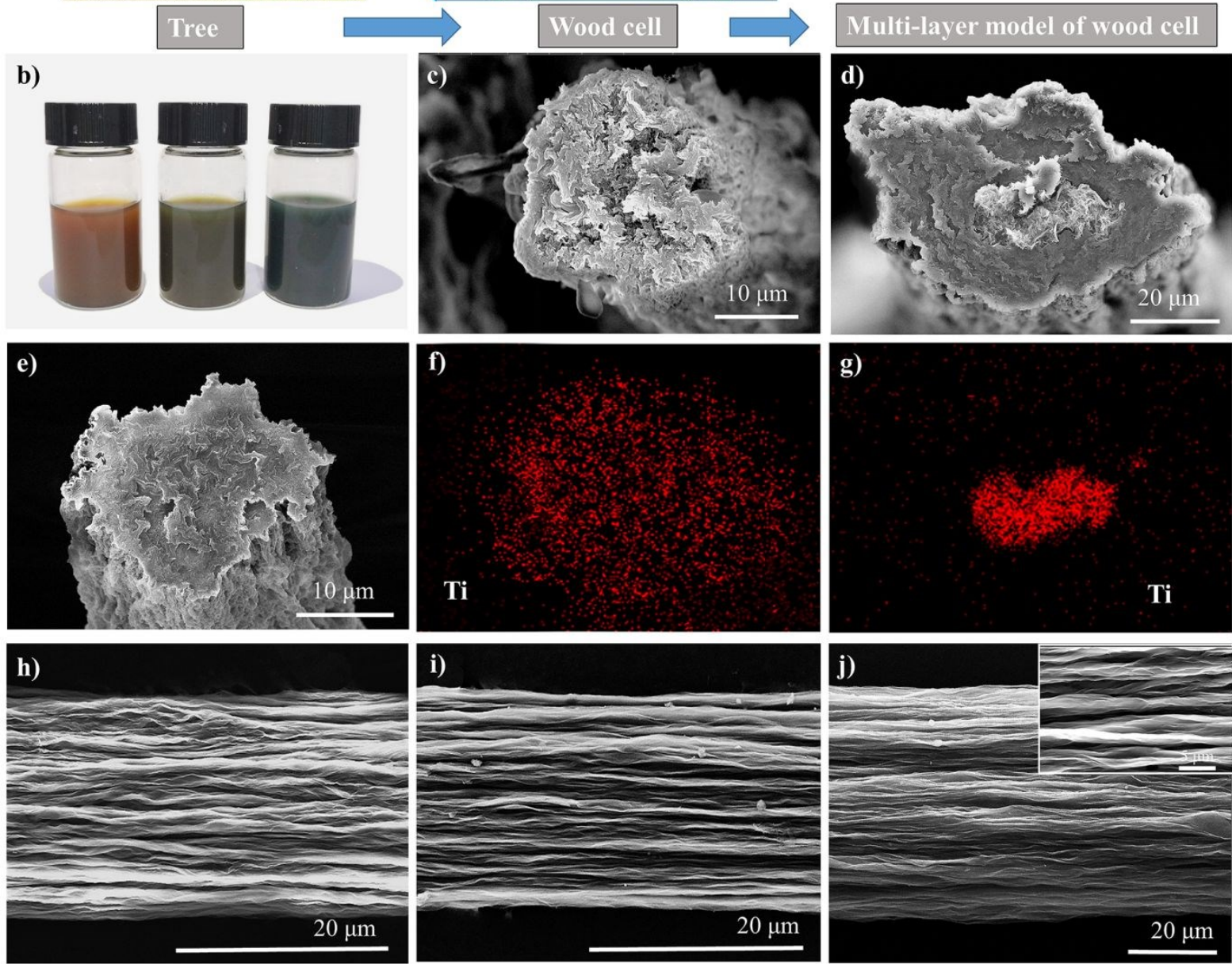

Fig. 2 (a) The microstructure of wood cell wall made up of a primary and three secondary layers (S1, S2 and S3), with the well-aligned cellulose microfibrils arranged in S2 layer, (b) Photo of a stable suspension of nanosheets in pure water $(12 \mathrm{mg} / \mathrm{mL})$, left to right: GO, MXene, MXene and GO with mass ration 1:1. Section morphology of (c) MX/GO fiber, (d) MX@GO fiber, (e) GO fiber. EDS mapping analysis of f) MX/GO fiber, (g) MX@GO fiber. Surface morphology of (h) GO fiber, (i) MX/GO fiber, (j) MX@GO fiber. 


\subsection{The mechanical property of MX@GO fiber}

The typical tensile strength-strain curves of GO, MX/GO and MX@GO fiber are shown in Fig. 3a. The MX/GO-1 fiber had a tensile strength of $140 \mathrm{MPa}$ at $0.95 \%$ ultimate elongation with an 18 GPa young's modulus. In comparison, the coaxial MX@GO fiber exhibited a tensile strength of $290 \mathrm{MPa}$ at $1.5 \%$ elongation with a $30 \mathrm{GPa}$ Young's modulus, which is about two times greater than that of MX/GO-1 fiber. It is well known that chemical etching MXene possesses many functional groups $(-\mathrm{OH},-\mathrm{O}$, and $-\mathrm{F})$, implying the chemical interactions between the functional groups of the MXene sheets and $\mathrm{Ca}^{2+}$ ions after coagulation. The XRD pattern of $\mathrm{Ca}^{2+}$ modified MXene shown an increased layer-to-layer distance compared with that of the MXene (Fig. S3), which demonstrates $\mathrm{Ca}^{2+}$ ions enter the gallery spaces between MXene sheets. For the spectrum of $\mathrm{Ca}^{2+}$ modified MXene, the $\mathrm{C}=\mathrm{O}$ and $\mathrm{C}-\mathrm{O}\left(1222 \mathrm{~cm}^{-1}\right)$ stretch intensities decreased, showing the functional groups coordinates to $\mathrm{Ca}^{2+}$ ions (Fig. S5). Compared with GO, the as-obtained MXene lacks the carboxyl group. Importantly, it has been demonstrated that cross-linking bridge through carboxylate chelates to $\mathrm{Ca}^{2+}$ ions is strong than bridges through either weak alkoxide or dative bonds from carbonyl and hydroxyl groups.[19] And the weak bonds can be removed by water rinsing. The $\mathrm{Ca}^{2+}$ modified MXene membrane showed lower tensile strength (3MPa, Fig. S6), verifying the weak cross-linking bridges. Although the introduction of MXene sheets may be a good scheme to fabricate highperformance fiber, the presence of MXene nanosheets hinder the combination of de-protonated GO sheets with $\mathrm{Ca}^{2+}$ ions. This fact results in forming a three-dimensional graphene structure instead of a dense structure, as shown in Fig. 3b. This particular structure increases the crosssectional area for calculating the tensile strength; thus, the mechanical property of $\mathrm{MX} / \mathrm{GO}$ hybrid fiber is lower than that of MX@GO fiber. More interesting, it is noteworthy that MX/GO-2 fiber possessed a tensile strength of $185 \mathrm{MPa}$ at $0.77 \%$ ultimate elongation with a $25 \mathrm{GPa}$ young's modulus. It shows that the mechanical strength tended to increase with the decrease of the MXene content. This fact indicates the existence of MXene reduces the mechanical property of MX/GO fiber (Fig. 3a). 

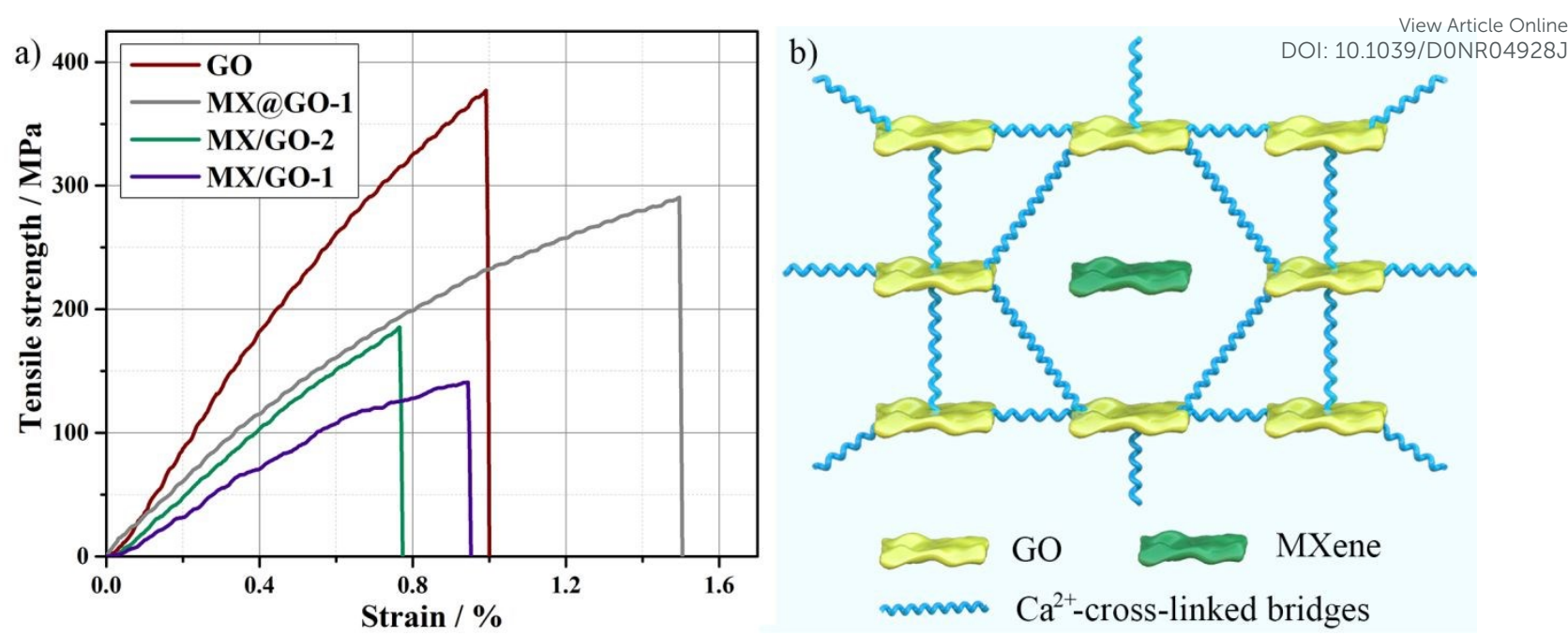

Fig. 3 (a) Typical mechanical measurements under tension for GO fiber, MX@GO-1 fiber and MX/GO fiber with different weight ratios, (b) Schematic illustration showing the interconnected model of MX/GO fiber.

Different from the MX/GO hybrid fiber, the ultimate performance of MX@GO fiber may be attributed to the support of the GO shell (mechanical layer). The performance of the distinct GO shell is associated with its self-assembly behavior and the inside orientation of nanosheets. For fibers prepared by wet spinning, the skin effect will inevitably occur during the flow-driven assembly process. The hierarchical microstructure is formed with well-aligned graphene sheets on fiber surfaces but random sheet alignments in graphene fiber.[20] Benefitting from the distinct core-shell structure, highly orientated and homogeneous GO sheets throughout the shell may be achieved by utilizing the double-tension effect of internal and external fluids to mitigate the skin effect.

To investigate the influence of the double-tension effect on the mechanical performance of MX@GO fiber, we synthesized the MX@GO-1 fiber by adjusting the velocity difference. It must be pointed out that the injection velocity of the MXene solution needs to be higher than that of the GO solution. The mechanical strength rose with the increasing the velocity difference of the MXene and GO solution. However, when the velocity of the MXene solution is lower than that of the GO solution, the tensile strength of MX@GO fiber is mainly determined by the velocity difference of the linear speed of the rotating coagulation bath and 


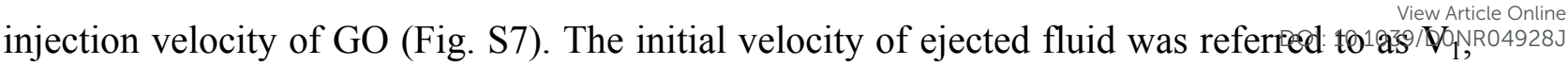
and the linear speed of the rotating coagulation bath at a special location was referred to as $\mathrm{V}_{2}$. As shown in Fig. 4a, with the increase of the velocity difference from 1 to 4.5 , the tensile strength of MX@GO fiber gradually rose. Notably, due to the much-improved sheet alignment order by adjusting the velocity difference $\left(4.5 \mathrm{~V}_{1}=\mathrm{V}_{2}\right)$, the diameter of the stretched MX@GO1 fiber could be lowered to about $56 \mu \mathrm{m}$. The MX@GO-1 fiber had a tensile strength of $290 \mathrm{MPa}$ at $1.5 \%$ ultimate elongation with a $30 \mathrm{GPa}$ Young's modulus. The measured mechanical properties are generally better compared with the neat graphene fiber reported previously.[21-25]

It means that, with the increase of the velocity difference, the double-tension effect will provide a larger stretching ratio at both sides of the GO sheath layer simultaneously. GO nanosheets were dynamically rearranged along the fiber direction. Thus the well-aligned GO layer shows improved mechanical properties. Compared with MX@GO fiber, MX/GO fiber could sustain a lower stretching ration (about 3). It results from the absence of a robust crosslinking bridge between the GO and MXene, leading to a lower alignment degree and the declined macroscopic mechanical properties. By contrast, after considering both solution viscosity and shear stress, GO and MXene dispersion was loaded in the syringe and ejected through two capillaries (inner diameter of $0.26 \mathrm{~mm}$ needle coaxially placed inside an inner diameter of 1.12 mm one) for the manufacture of MX@GO fiber without the double-tension effect. The large diameter capillary $(1.12 \mathrm{~mm})$ allowed the fabrication of graphene fiber with a lower alignment degree, leading to low tensile strength (60 MPa). This indicates that a high drawing ratio is essential to obtain highly aligned nanosheets and underlines the contribution of the double-tension effect to the mechanical property of the coaxial fiber. Observed increases in strength can be attributed to improved nanosheets alignment in the as-obtained fiber.[26] There is now extensive evidence of nanosheets orientation in as-fabricated fiber if they experience a high draw ratio before coagulated into a fibrillar form.[20, 27]

Additionally, the thickness of the GO shell is easily adjusted by changing the concentration. It provides a way to design the mechanical property of MX@GO fiber as needed 
freely. We have carried out a detailed study to evaluate the contribution Dof 1 s ${ }^{\text {Vef }}$ View Article Online concentration to the mechanical properties of MX@GO fiber based on the high mechanical stretching ratio. As shown in Fig. 4b, when the mass ratio decreased from 3 to 0 , the thickness of the GO shell gradually increased, and the tensile strength of MX@GO fiber rose from around $90 \mathrm{MPa}$ to $377 \mathrm{MPa}$. It is concluded that the GO shell determines the strength of the coaxial fiber, which can be easily controlled by adjusting the concentration of GO solution. These results support our hypothesis that the strength of MX@GO fiber is depends on its shell thickness and the inside nanosheets orientation distribution. Consequently, the MXene core will also be expected to play a vital role in the conductivity of the coaxial fiber.
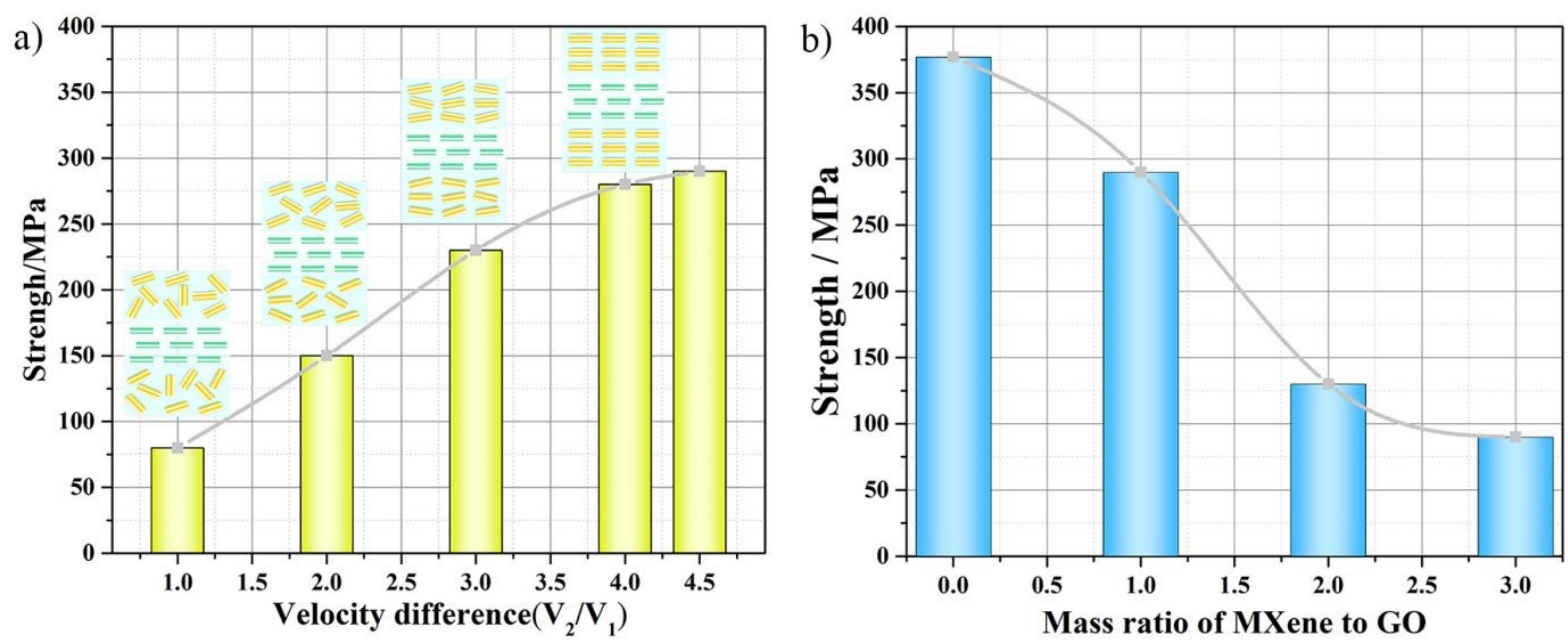

Fig. 4 Mechanical properties of MX@GO fiber concerning (a) velocity difference and (b) mass ratio.

\subsection{The electrical conductivity of MX@GO fiber}

Inspired by the function of the inner cell wall S3 layer (as a transport channel of water and other nutrients), the MXene layer can be designed as an electron transport path to improve the electrical property of the MX@GO fiber. The conductivity can be easily adjusted by changing the solution concentration. As mentioned previously, when the mass ratio increased from 0 to 3, the GO sheath layer's thickness decreased gradually, and the tensile strength declined from around $377 \mathrm{MPa}$ to $90 \mathrm{MPa}$. As shown in Fig. 5a, with the increase in the concentration of the 
MXene solution, the electrical conductivity progressively increased from around 0 S $/ \mathrm{m}^{\mathrm{m}} \mathrm{Ot}$ - View Article Online $\mathrm{S} / \mathrm{m}$, which substantiates the electrical transfer path was formed and the diameter of the conductive core (MXene) rose with the increasing mass ratio. It demonstrates that the MXene core significantly determines the electrical property of the biomimetic coaxial fiber, and the desired electricity can be achieved by optimizing the concentration of MXene solution.

In contrast with the MX/GO fiber, the high conductivity of the MX@GO fiber can be explained as follows. When coaxial fluid ejects into the rotating coagulation bath, the GO nanosheets quickly solidify and then yield a well-aligned core-shell structure without hybrid. On the other hand, with the MX@GO-1 fiber drying, the pores made up of MXene nanosheets near contact with each other so that the conductivity increased with the increase of the contact area. Unfortunately, the MX@GO-0.5 fiber was electrically insulating similar to neat GO fiber. The low conductivity of MX@GO-0.5 fiber may result from a disconnected electron transport path at high stretching rates. Though the MX@GO-0.5 fiber possessed a very low conductivity, MX/G0-0.5 fiber exhibited a high tensile strength of $330 \mathrm{MPa}$. Fortunately, with increasing mass ratio of MXene to GO, the MXene core could provide highways for the transport of electrons.

Additionally, the introduction of MXene enables the MX@GO fiber to have high electrical conductivity but makes the composite fiber electrically insulating unfortunately. The reason comes from the fact that GO fiber is typically electrically insulating due to the presence of defects in the lattice structure and many oxygen functional groups, which is consistent with previous works.[28] Furthermore, MXene sheets do not form a continuous system in MX/GO fiber so that the electrons transfer slower between the GO sheets and MXene sheets (Fig. 3b). This lousy result indicates that the introduction of MXene sheets into GO fiber is not an effective way to improve the electrical conductivity of hybrid fiber. Overall, these results confirm our hypothesis that GO shell can be used as mechanical support to assemble MXene into macro materials. The MXene core can serve as the electric transfer path to improve the conductivity of MX@GO fiber.

An optimized balance between mechanical and electrical properties should be achieved to 
design a high-performance fiber. As expected, the mechanical conductivity tended $q b^{1}{ }^{1}$ View Article Online while the electrical properties declined with the decreasing mass ratio of MXene to GO, as shown in Fig. 5a. The measured peak tensile strength and electrical conductivity rose to 377 MPa and $4600 \mathrm{~S} / \mathrm{m}$, respectively. Therefore, to balance the high mechanical and electrical property, the MX@GO-1 was chosen to investigate the thermal stability. Moreover, the MX@GO-1 fiber possessed high tensile strength (290 MPa) and electrical conductivity (2400 $\mathrm{S} / \mathrm{m}$ ), representing improvements of $107 \%$ and $100 \%$, respectively, as compared to those of the $\mathrm{MX} / \mathrm{rGO}$ hybrid fiber.

Conclusively, inspired by the structure of wood, the wood-like core-shell MX@GO fiber with super performance was successfully obtained. The improved mechanical and electrical properties are attributed to the GO sheath layer and MXene core layer, respectively.
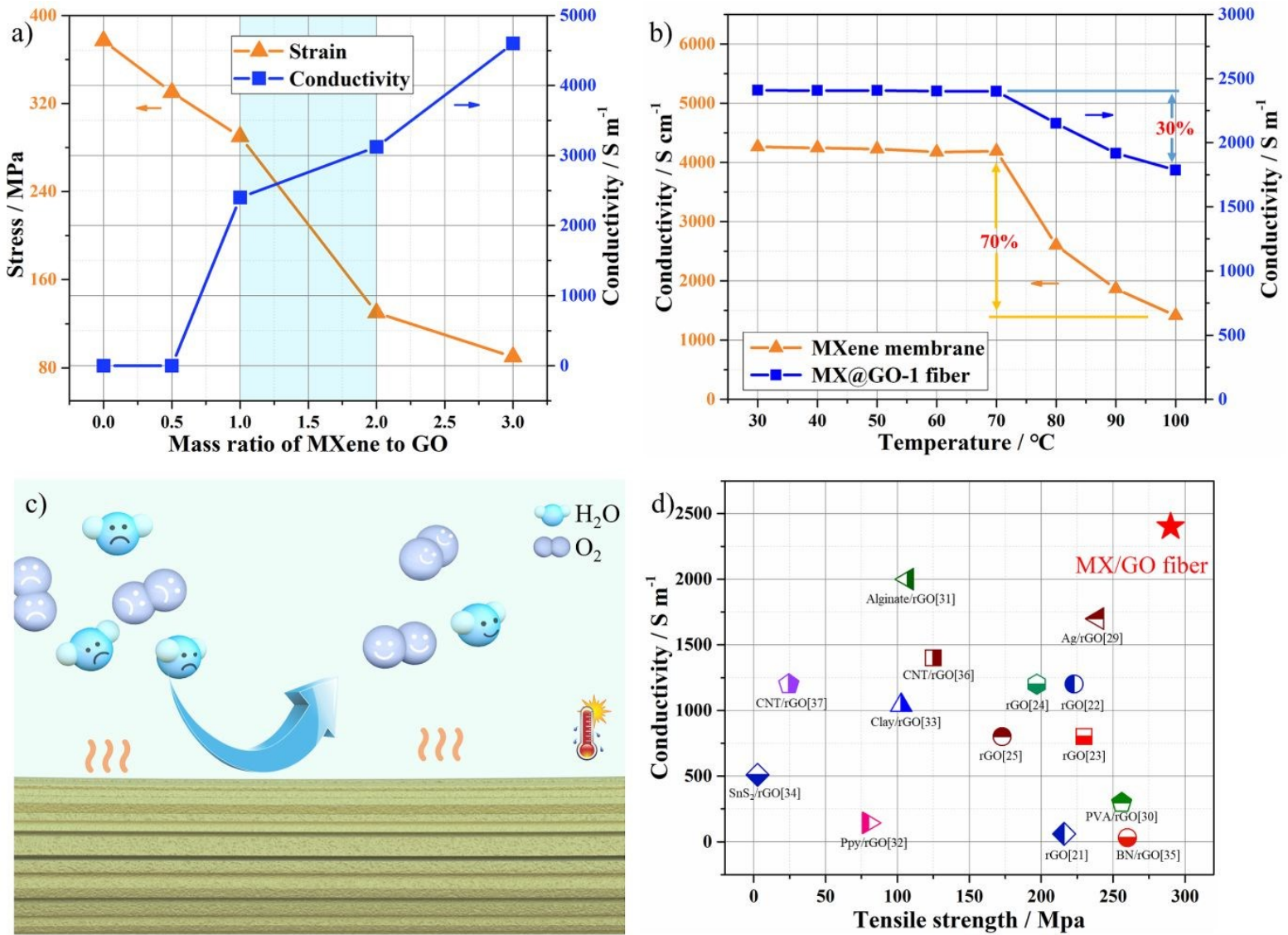

Fig. 5 (a) Electrical and mechanical properties for MX@GO fiber concerning mass ratio, (b) Conductivity of MXene membrane and MX@GO-1 fiber from room temperature to $100^{\circ} \mathrm{C}$, (c) Schematic illustration showing the heating protection model, (d) Comparison of tensile strength and conductivity of GO-based fiber with literature.[21-25, 29-37] 


\subsection{Thermal stability of MX@GO fiber}

To promote MXene-based materials in a broader range of fields, the thermal stability of MXene-based materials must be investigated. Fig. $5 \mathrm{~b}$ shows the conductivity measurements of MXene membrane after different temperature processes, and the conductivity curve can be divided into two stages. At low temperature, the electrical conductivity almost remains constant with the increase of temperature until $70{ }^{\circ} \mathrm{C}$, and the measured peak electrical conductivity of MXene membrane was up to $4260 \mathrm{~S} / \mathrm{cm}$. Then, the conductivity sharply decreased when the temperature was from 70 to $100{ }^{\circ} \mathrm{C}$, which means the MXene membrane might be oxidized.

Additionally, the conductivity of MXene membrane decreased to $1400 \mathrm{~S} / \mathrm{cm}$ with a reduction of about $70 \%$ at $100^{\circ} \mathrm{C}$, which will limit their application. Like with the neat MXene membrane, the conductivity of MX@GO-1 fiber remained stable with the increase of temperature until $70{ }^{\circ} \mathrm{C}$. The measured peak electrical conductivity was up to $2400 \mathrm{~S} / \mathrm{m}$. Then, the conductivity of MX@GO-1 fiber declined with the increase of temperature from 70 to $100^{\circ} \mathrm{C}$. More interesting, the conductivity of MX@GO-1 fiber only decreased to $1800 \mathrm{~S} / \mathrm{m}$ with a reduction of about $30 \%$ at $100^{\circ} \mathrm{C}$. The reason for the decrement may be the presence of an oxide element in the GO layer. The conductivity of biomimetic MX@GO fiber only had a light decay in contrast with the neat MXene membrane. It could keep stable for a long time (Fig. S8), proving the excellent thermal stability of GO-wrapped MXene sheets. The good thermal stability of the MX@GO fiber can be explained as follows: Due to the low thermal conductivity of GO, the GO layer can also be used as a functional layer to decelerate the heat propagation velocity.

On the other hand, the MXene core is warped tightly by GO sheath without any gaps and voids. The dense GO sheath prevents the exposure of the MXene sheets to air condition, which is extremely important for improving the thermal stability of MX@GO fiber. How the structure and properties of MXene change with temperature will be revealed in our follow-up work. Additionally, Fig. 5d presents a comparison of the optimized mechanical and electrical 
properties achieved in this study with the values reported in the literature. The Deorimparifisont R04928J authenticates the superior performance of the as-fabricated biomimetic fiber compared with those in the literature. In addition to excellent mechanical and electrical performance, the wood-like core-shell fiber possesses outstanding electromagnetic interference shielding property of $70 \mathrm{~dB}$ for the MX@GO-1 fiber (Fig. S9). Therefore, this scalable, cost-effective, and continuous wet-spinning technique can achieve the high-performance coaxial fiber. In summary, benefitting from the excellent thermal stability of the GO@GO fiber, biomimetic MX@GO fiber will have a more extensive practical application in wearable electronic devices.

\section{Conclusions}

In summary, inspired by the wood structure, we successfully developed a facile and cost effective method to fabricate a wood-like MX@GO fiber for the first time. It delivers high tensile strength (290 MPa), electrical conductivity $(2400 \mathrm{~S} / \mathrm{m})$, and good temperature resistance $\left(1800 \mathrm{~S} / \mathrm{m}\right.$ with a reduction of about $30 \%$ at $\left.100^{\circ} \mathrm{C}\right)$, which represents improvements of $133 \%$ as compared to those of the MXene membrane. The highly conductive MX@GO-1 fiber presents an excellent EMI SE of about $70 \mathrm{~dB}$ over the whole X-band frequency range. In contrast with the MX/GO fiber, the mechanical and electrical properties of biomimetic fiber could be freely designed as needed without any chemical post-treatment. This approach is simple, industrially viable, and general for another conductive particle that cannot be wet-spun continuous by previous approaches, which paves the way to fabricate high-performance fiber with the desired function and structure further to meet the requirements of sustainable development.

\section{Acknowledgments}

This research is supported by "National Natural Science Foundation of China" (No. 11902256), "Natural Science Basic Research Program of Shaanxi" (No.2019JQ-479) and the China Scholarship Council.

\section{Author Contributions}

The manuscript was written through the contributions of all authors. All authors have 
approved to the final version of the manuscript

\section{Conflict of Interests}

The authors declare that there is no conflict of interest regarding the publication of this paper.

\section{Reference}

[1] M. Naguib, M. Kurtoglu, V. Presser, J. Lu, J. Niu, M. Heon, L. Hultman, Y. Gogotsi, M.W. Barsoum, Two - dimensional nanocrystals produced by exfoliation of Ti3AlC2, Adv. Mater. 23(37) (2011) 4248-4253.

[2] W.-T. Cao, F.-F. Chen, Y.-J. Zhu, Y.-G. Zhang, Y.-Y. Jiang, M.-G. Ma, F. Chen, Binary strengthening and toughening of MXene/cellulose nanofiber composite paper with nacre-inspired structure and superior electromagnetic interference shielding properties, ACS Nano 12(5) (2018) 4583-4593.

[3] L. Zhao, B. Dong, S. Li, L. Zhou, L. Lai, Z. Wang, S. Zhao, M. Han, K. Gao, M. Lu, Interdiffusion Reaction-Assisted Hybridization of Two-Dimensional Metal-Organic Frameworks and Ti3C2Tx Nanosheets for Electrocatalytic Oxygen Evolution, ACS Nano 11(6) (2017) 5800-5807.

[4] T. Zhou, C. Wu, Y. Wang, A.P. Tomsia, M. Li, E. Saiz, S. Fang, R.H. Baughman, L. Jiang, Q. Cheng, Super-tough MXene-functionalized graphene sheets, Nat. Commun. 11(1) (2020) 1-11.

[5] Z. Chen, Y. Hu, H. Zhuo, L. Liu, S. Jing, L. Zhong, X. Peng, R.-c. Sun, Compressible, Elastic, and Pressure-Sensitive Carbon Aerogels Derived from 2D Titanium Carbide Nanosheets and Bacterial Cellulose for Wearable Sensors, Chem. Mater. 31(9) (2019) 33013312 .

[6] Y. Ma, Y. Yue, H. Zhang, F. Cheng, W. Zhao, J. Rao, S. Luo, J. Wang, X. Jiang, Z. Liu, 3D synergistical MXene/reduced graphene oxide aerogel for a piezoresistive sensor, ACS Nano 12(4) (2018) 3209-3216.

[7] J. Liu, H.B. Zhang, X. Xie, R. Yang, Z. Liu, Y. Liu, Z.Z. Yu, Multifunctional, superelastic, and lightweight MXene/polyimide aerogels, Small 14(45) (2018) 1802479.

[8] U.G. Wegst, H. Bai, E. Saiz, A.P. Tomsia, R.O. Ritchie, Bioinspired structural materials, Nat. Mater. 14(1) (2015) 23-36.

[9] L.-B. Mao, H.-L. Gao, H.-B. Yao, L. Liu, H. Cölfen, G. Liu, S.-M. Chen, S.-K. Li, Y.X. Yan, Y.-Y. Liu, Synthetic nacre by predesigned matrix-directed mineralization, Science 354(6308) (2016) 107-110.

[10] Z.-L. Yu, N. Yang, L.-C. Zhou, Z.-Y. Ma, Y.-B. Zhu, Y.-Y. Lu, B. Qin, W.-Y. Xing, T. Ma, S.-C. Li, Bioinspired polymeric woods, Science advances 4(8) (2018) eaat7223.

[11] M. Liu, S. Wang, L. Jiang, Nature-inspired superwettability systems, Nature Reviews Materials 2(7) (2017) 1-17.

[12] L.J. Gibson, The hierarchical structure and mechanics of plant materials, J. Royal 
Soc. Interface 9(76) (2012) 2749-2766.

[13] L. Zorzetto, D. Ruffoni, Wood - Inspired 3D - Printed Helical Composites with Tunable and Enhanced Mechanical Performance, Adv. Funct. Mater. 29(1) (2019) 1805888.

[14] K.S. Novoselov, A.K. Geim, S.V. Morozov, D. Jiang, Y. Zhang, S.V. Dubonos, I.V. Grigorieva, A.A. Firsov, Electric field effect in atomically thin carbon films, Science 306(5696) (2004) 666-669.

[15] D.C. Marcano, D.V. Kosynkin, J.M. Berlin, A. Sinitskii, Z. Sun, A. Slesarev, L.B. Alemany, W. Lu, J.M. Tour, Improved synthesis of graphene oxide, ACS Nano 4(8) (2010) 4806-4814.

[16] Z. Xu, H. Sun, X. Zhao, C. Gao, Ultrastrong fibers assembled from giant graphene oxide sheets, Adv. Mater. 25(2) (2013) 188-193.

[17] L. Kou, C. Gao, Bioinspired design and macroscopic assembly of poly (vinyl alcohol)-coated graphene into kilometers-long fibers, Nanoscale 5(10) (2013) 4370-4378.

[18] J. Liu, H.B. Zhang, R. Sun, Y. Liu, Z. Liu, A. Zhou, Z.Z. Yu, Hydrophobic, flexible, and lightweight MXene foams for high - performance electromagnetic - interference shielding, Adv. Mater. 29(38) (2017) 1702367.

[19] S. Park, K.-S. Lee, G. Bozoklu, W. Cai, S.T. Nguyen, R.S. Ruoff, Graphene oxide papers modified by divalent ions - enhancing mechanical properties via chemical cross-linking, ACS Nano 2(3) (2008) 572-578.

[20] G. Xin, W. Zhu, Y. Deng, J. Cheng, L.T. Zhang, A.J. Chung, S. De, J. Lian, Microfluidics-enabled orientation and microstructure control of macroscopic graphene fibres, Nat. Nanotechnol. 14(2) (2019) 168-175.

[21] I.H. Kim, T.H. Im, H.E. Lee, J.S. Jang, H.S. Wang, G.Y. Lee, I.D. Kim, K.J. Lee, S.O. Kim, Janus Graphene Liquid Crystalline Fiber with Tunable Properties Enabled by Ultrafast Flash Reduction, Small 15(48) (2019) 1901529.

[22] L. Sheng, T. Wei, Y. Liang, L. Jiang, L. Qu, Z. Fan, Ultra-high toughness all graphene fibers derived from synergetic effect of interconnected graphene ribbons and graphene sheets, Carbon 120 (2017) 17-22.

[23] T. Ma, H.L. Gao, H.P. Cong, H.B. Yao, L. Wu, Z.Y. Yu, S.M. Chen, S.H. Yu, A Bioinspired Interface Design for Improving the Strength and Electrical Conductivity of Graphene - Based Fibers, Adv. Mater. 30(15) (2018) 1706435.

[24] D. Yu, K. Goh, H. Wang, L. Wei, W. Jiang, Q. Zhang, L. Dai, Y. Chen, Scalable synthesis of hierarchically structured carbon nanotube-graphene fibres for capacitive energy storage, Nat. Nanotechnol. 9(7) (2014) 555.

[25] Y. Zhao, C. Jiang, C. Hu, Z. Dong, J. Xue, Y. Meng, N. Zheng, P. Chen, L. Qu, Large-scale spinning assembly of neat, morphology-defined, graphene-based hollow fibers, ACS Nano 7(3) (2013) 2406-2412.

[26] G. Xin, T. Yao, H. Sun, S.M. Scott, D. Shao, G. Wang, J. Lian, Highly thermally conductive and mechanically strong graphene fibers, Science 349(6252) (2015) 1083-1087.

[27] H. Park, K. Lee, Y. Kim, S. Ambade, S. Noh, W. Eom, J. Hwang, W. Lee, J. Huang, T.H. Han, Dynamic assembly of liquid crystalline graphene oxide gel fibers for ion transport, Science advances 4(11) (2018) eaau2104. 
[28] Z. Xu, C. Gao, Graphene chiral liquid crystals and macroscopic assembbled. fivibriesticle Online Nat. Commun. 2(1) (2011) 1-9.

[29] S.S. Yoon, K.E. Lee, H.-J. Cha, D.G. Seong, M.-K. Um, J.-H. Byun, Y. Oh, J.H. Oh, W. Lee, J.U. Lee, Highly conductive graphene/Ag hybrid fibers for flexible fiber-type transistors, Scientific reports 5 (2015) 16366.

[30] S. Chen, W. Ma, H. Xiang, Y. Cheng, S. Yang, W. Weng, M. Zhu, Conductive, tough, hydrophilic poly (vinyl alcohol)/graphene hybrid fibers for wearable supercapacitors, J. Power Sources 319 (2016) 271-280.

[31] X. Hu, M. Tian, N. Pan, B. Sun, Z. Li, Y. Ma, X. Zhang, S. Zhu, Z. Chen, L. Qu, Structure-tunable graphene oxide fibers via microfluidic spinning route for multifunctional textile, Carbon 152 (2019) 106-113.

[32] X. Ding, Y. Zhao, C. Hu, Y. Hu, Z. Dong, N. Chen, Z. Zhang, L. Qu, Spinning fabrication of graphene/polypyrrole composite fibers for all-solid-state, flexible fibriform supercapacitors, Journal of Materials Chemistry A 2(31) (2014) 12355-12360.

[33] G. Chen, Y. Ai, I.T. Mugaanire, W. Ma, B.S. Hsiao, K. Hou, M. Zhu, A simple inorganic hybrids strategy for graphene fibers fabrication with excellent electrochemical performance, J. Power Sources 450 (2020) 227637.

[34] Z. Ren, J. Wen, W. Liu, X. Jiang, Y. Dong, X. Guo, Q. Zhao, G. Ji, R. Wang, N. Hu, Rational Design of Layered SnS 2 on Ultralight Graphene Fiber Fabrics as Binder-Free Anodes for Enhanced Practical Capacity of Sodium-Ion Batteries, Nano-Micro Letters 11(1) (2019) 66.

[35] C.S. Boland, S. Barwich, U. Khan, J.N. Coleman, High stiffness nano-composite fibres from polyvinylalcohol filled with graphene and boron nitride, Carbon 99 (2016) 280288.

[36] Y. Sun, Y. Wang, C. Hua, Y. Ge, S. Hou, Y. Shang, A. Cao, Water-responsive helical graphene-oxide fibers incorporating a continuous carbon nanotube network, Carbon 132 (2018) 394-400.

[37] H. Cheng, Z. Dong, C. Hu, Y. Zhao, Y. Hu, L. Qu, N. Chen, L. Dai, Textile electrodes woven by carbon nanotube-graphene hybrid fibers for flexible electrochemical capacitors, Nanoscale 5(8) (2013) 3428-3434. 


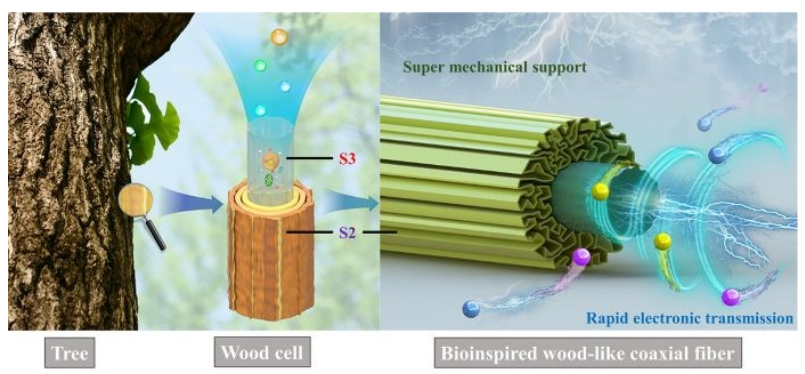

Inspired by the structure of wood, a coaxial MXene@GO fiber with a superior mechanical shell and electrical core is fabricated. 